\title{
Should we consider heparin prophylaxis in COVID-19 patients? a systematic review and meta-analysis
}

\author{
Mohamed Abdel-Maboud ${ }^{1,7}$ (1) $\cdot$ Amr Menshawy ${ }^{1} \cdot$ Ahmed Elgebaly $^{2} \cdot$ Eshak I. Bahbah $^{3} \cdot$ Gehad El Ashal $^{4}$. \\ Ahmed Negida ${ }^{5,6}$
}

Accepted: 10 August 2020 / Published online: 20 August 2020

(c) Springer Science+Business Media, LLC, part of Springer Nature 2020

\section{To the editor,}

The COVID-19 pandemic has affected more than 21 million individuals around the world, with over 750,000 deaths. A large body of evidence suggests that COVID-19 is associated with coagulopathy and induced hypercoagulation. Major thromboembolic events were found in postmortem studies of COVID-19 patients [1]. Likewise, a recent study found a possible anti-inflammatory role of heparin prophylaxis lowering the levels of interleukin-6-which play a role in the cytokine storm of severe COVID-19 [2].

Therefore, some experts recommend prophylactic heparin to prevent thromboembolic events in COVID-19 [3, 4]. On the other hand, heparin might induce thrombocytopenia and increase the mortality risk rather than improving patients' outcomes. Still, it is unclear whether prophylactic heparin is associated with decreased COVID-19 mortality. Because patients with COVID-19 show paradoxical incidence of thrombotic events along with bleeding events, it is not clear

Electronic supplementary material The online version of this article (https://doi.org/10.1007/s11239-020-02253-x) contains supplementary material, which is available to authorized users.

Mohamed Abdel-Maboud

MohamedAbdel-Maboud.6.206@azhar.edu.eg

Faculty of Medicine, Al-Azhar University, Cairo, Egypt

2 Department of Clinical Pathology, Faculty of Medicine, Al-Azhar University, Cairo, Egypt

3 Faculty of Medicine, Al-Azhar University, New Damietta, Egypt

4 Ministry of Health Hospitals, Cairo, Egypt

5 Zagazig University Hospitals, Zagazig University, Sharkia, Egypt

6 School of Pharmacy and Biomedical Sciences, University of Portsmouth, Portsmouth, UK

7 Al-Hussein University Hospital, Cairo, Egypt whether a strategy of low-dose or full-dose prophylactic anticoagulants would result in net benefit or harm.

To answer this question, we conducted this meta-analysis to investigate whether prophylactic heparin use is associated with lower. The detailed methods of systematic literature search, screening, selection, data extraction, and data analysis are detailed in the supplementary file no.1.

We included eight studies ( $n=2946$ patients), summarized in the supplementary file Table 1 . Three studies were conducted in China, one study in United States, one study in Italy, one in Brazil, one in the Netherlands, and one in Spain. The age of the included patients ranged from 39 to 85 years. Seventy percent of the included cohort were males, and $18 \%$ had suffered from comorbidities. Of them, five studies were included in the meta-analysis with a total of 2772 patients (prophylactic heparin group $n=2006$ patients vs. non-prophylactic heparin group $\mathrm{n}=766$ patient.

Of our pertaining cohort, $72 \%$ of the COVID-19 patients received prophylactic heparin $(2.5 \%$ were UFH) during their treatment course. The pooled risk ratio of mortality did not favor either of the two groups (RR 0.96, with 95\% CI from 0.807 to 1.147 ; Fig. 1a).

Similarly, prophylactic heparin was not associated with significant reductions or increases in the mortality rates in moderate COVID-19 patients (RR 0.87, with 95\% CI from 0.69 to 1.10 ) or severe COVID-19 patients (RR 1.09, with $95 \%$ CI from 0.835 to 1.433 ).

Meanwhile, dosages ranged from 40 to $60 \mathrm{mg}$ or 4000-8000 IU b.i.d-depending on the BMI, some reports included an additional subgroup of 10,000-15,000 U/day. To differentiate between low prophylactic dose and high dose used for prophylaxis: we performed a further subgroup analysis (supplementary file; Fig. 1), which revealed no significant difference.

These findings come against several preliminary reports suggesting the potential role of heparin in reducing mortality [5]. Meanwhile, regression models (Fig. 1b) revealed no 


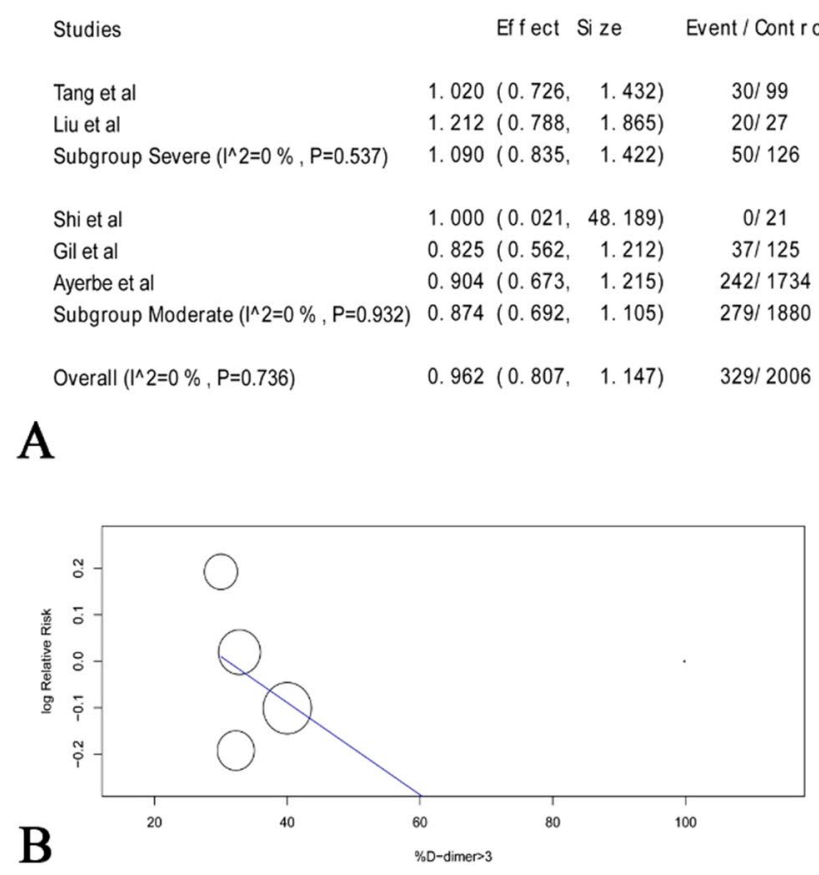

Fig. 1 a Shows a forest plot of the pooled risk ratio of mortality between the prophylactic heparin and non-prophylactic heparin groups with the corresponding $95 \%$ confidence intervals. b Shows the

significant effect of age, sex, comorbidities or co-interventions $(\mathrm{P}=0.3,0.2,0.6,0.4$; respectively). However, interaction regressions of combined severity, D-dimer $>3 \mu \mathrm{g} / \mathrm{L}$, platelet count $>100 \times 10^{9} / \mathrm{L}$ and $\mathrm{PT}<14 \mathrm{~s}$ were approximate to significance $(\mathrm{P}=0.05)$.

High expression of cytokines in response to severe lung inflammation and endothelial damage could explain the hypercoagulable state in COVID-19 patients [6]. Elevated D-dimer levels in COVID-19 patients might result from the upregulation of intrinsic fibrinolysis cascades in the lung and cleaved in the blood. Gaertner and Massberg hypothesize a bidirectional relationship between thrombin formation and cytokines upregulation [7].

Additionally, heparin has an anti-inflammatory effect that may also be pertinent in this activity. Several reports were illustrating this anti-inflammatory property, and one of the described mechanisms proposes the attachment of heparin to the pro-inflammatory cytokines. In turn, this significantly inhibits neutrophil chemotaxis and leukocytes migration. Also, positively charged peptides complement factors are neutralized, and the acute phase proteins are cloistered [8]. A systematic literature review reported that heparin could reduce the pro-inflammatory cytokines, but this evidence was based on preliminary data with limited sample sizes [9]. Tang et al. [5] suggested that certain patients might benefit from heparin prophylaxis. They reported that patients with sepsis index score $\geq 4$ or elevated D-dimer level $>3 \mathrm{ug} / \mathrm{L}$ had lower mortality with heparin prophylaxis [5].
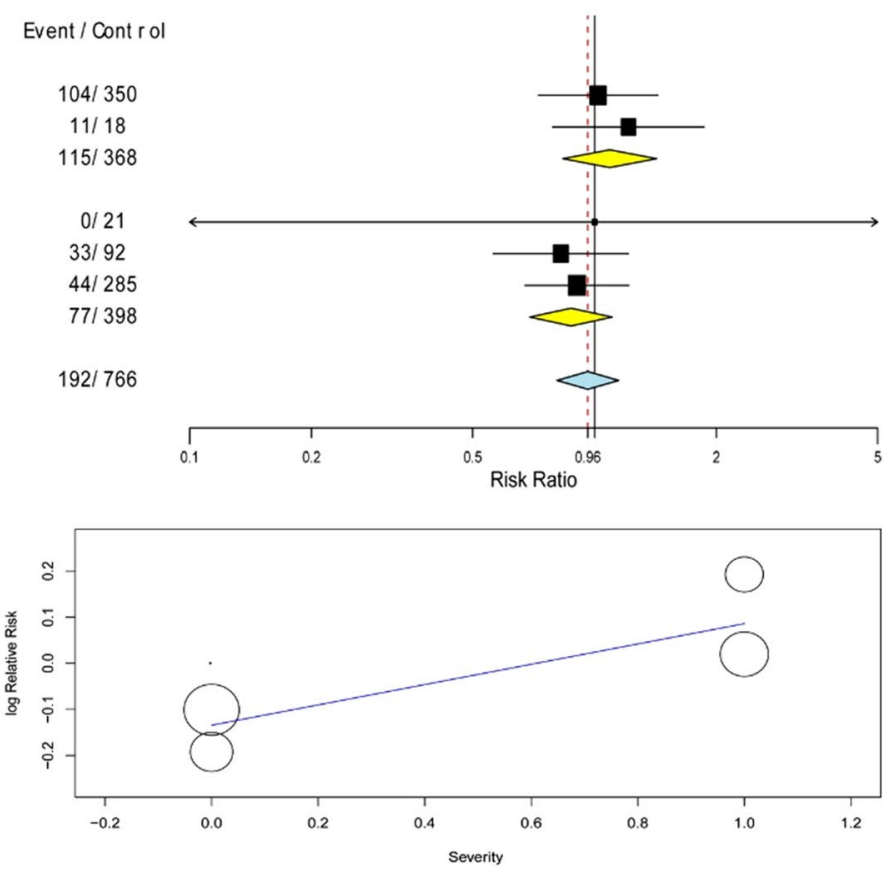

overall meta-regression risk ratio of the interaction between severity/ D-dimer on $\mathrm{x}$-axis and mortality on $\mathrm{y}$-axis

To the best of our knowledge, this is the first meta-analysis to investigate whether patients receiving prophylactic heparin had lower mortality. It is worth notice that most of the included studies were retrospective studies with small sample size, which potentially encompass selection bias and confounding bias.

In conclusion, current evidence is not sufficient to support the role of prophylactic heparin in reducing mortality among COVID-19 patients. However, the positive effect of prophylactic heparin seems to favor patients of moderate symptoms with a combined D-dimer $>3 \mu \mathrm{g} / \mathrm{L}$, a platelet count $>100 \times 10^{9} / \mathrm{L}$, and a PT $<14 \mathrm{~s}$; regardless of comorbidity, sex or age. We recommend further randomized-controlled trials with patient stratification according to D-dimer levels, PT and platelet count.

Funding None (authors confirm they did not receive any funding to do this work).

\section{Compliance with ethical standards}

Conflict of interest All authors confirm no financial or personal relationship with a third party whose interests could be positively or negatively influenced by the article's content. 


\section{References}

1. Wichmann D, Sperhake J-P, Lütgehetmann M, Steurer S, Edler C, Heinemann A et al (2020) Autopsy findings and venous thromboembolism in patients with COVID-19. Ann Intern Med. https:// doi.org/10.7326/M20-2003

2. Shi C, Wang C, Wang H, Yang C, Cai FEI, Zeng F et al (2020) The potential of low molecular weight heparin to mitigate cytokine storm in severe covid-19 patients: a retrospective clinical study. medRxiv. https://doi.org/10.1101/2020.03.28.20046144

3. Lillicrap D (2020) Disseminated intravascular coagulation in patients with 2019-nCoV pneumonia. J Thromb Haemost 18:786-787

4. Thachil J (2020) The versatile heparin in COVID-19. J Thromb Haemost 18(5):1020-1022

5. Tang N, Bai H, Chen X, Gong J, Li D, Sun Z (2020) Anticoagulant treatment is associated with decreased mortality in severe coronavirus disease 2019 patients with coagulopathy. J Thromb Haemost 18(5):1094-1099
6. Xiong TY, Redwood S, Prendergast B, Chen M (2020) Coronaviruses and the cardiovascular system: acute and long-term implications. Eur Heart J 41(19):1798-1800. https://doi.org/10.1093/ eurheartj/ehaa231

7. Gaertner F, Massberg S (2016) Blood coagulation in immunothrombosis - at the frontline of intravascular immunity. In: Seminars in immunology, vol 28. Academic Press, London, pp 561-569

8. Mousavi S, Moradi M, Khorshidahmad T, Motamedi M (2015) Anti-inflammatory effects of heparin and its derivatives: a systematic review. Adv Pharmacol Sci 2015:507151. https://doi. org/10.1155/2015/507151

9. Li JP, Vlodavsky I (2009) Heparin, heparan sulfate and heparanase in inflammatory reactions. Thromb Haemost 102(5):823-828

Publisher's Note Springer Nature remains neutral with regard to jurisdictional claims in published maps and institutional affiliations. 